\title{
Dietary patterns and the risk of cardiovascular disease and mortality: a population-based cohort study of older British men
}

\author{
J. L. Atkins ${ }^{1}$, P. H. Whincup ${ }^{2}$, R. W. Morris ${ }^{1}$, L. T. Lennon ${ }^{1}$, O. Papacosta ${ }^{1}$ and S. G. Wannamethee ${ }^{1}$ \\ ${ }^{1}$ Department of Primary Care and Population Health, University College London, London, NW3 $2 P F$ and ${ }^{2}$ Population \\ Health Research Centre, Division of Population Health Sciences and Education, St George's, University of London, \\ London, SW17 ORE, UK
}

Although diet quality is implicated in cardiovascular disease (CVD) risk, few studies have investigated the relationship between overall diet quality and the risk of CVD and mortality in older adults in Great Britain ${ }^{(1)}$. The Elderly Dietary Index (EDI) was developed to address adherence to nutritional recommendations for older adults, based on the frequency of consumption of specific foods/food groups $^{(2)}$. However, the EDI has not been compared to other widely used dietary indices such as the Healthy Diet Indicator (HDI), based on the World Health Organization's dietary guidelines for intake of nutrients and food components ${ }^{(3)}$. The aim of this study was to prospectively examine associations between dietary patterns (HDI and EDI) and risk of CVD and all-cause mortality in older British men.

4252 older men (aged 60-79 years) from the British Regional Heart Study, were followed-up for a mean period of 11.3 years for cardiovascular morbidity and mortality. Baseline food frequency questionnaire data were used to generate two overall dietary scores HDI and EDI, with higher scores indicating greater compliance with dietary recommendations. Cox proportional hazards regression analyses assessed associations between tertiles of HDI/EDI and risk of all-cause mortality, CVD mortality, CVD events and coronary heart disease (CHD) events, adjusting for age, smoking, alcohol, physical activity, social class, body mass index and energy intake.

\begin{tabular}{|c|c|c|c|c|c|}
\hline & & \multicolumn{3}{|c|}{ Elderly Dietary Index (Tertiles) } & \multirow[b]{2}{*}{$P$-value for trend } \\
\hline & & Low (4-25 points) & Medium (26-28 points) & High $29-36$ points & \\
\hline All-cause mortality & $\begin{array}{l}\text { Model } 1 \\
\text { Model } 2\end{array}$ & $\begin{array}{l}1.00 \\
1.00\end{array}$ & $\begin{array}{l}0.82(0.71-0.95)^{*} \\
0.92(0.79-1.07)\end{array}$ & $\begin{array}{l}0.65(0.56-0.76)^{*} \\
0.80(0.67-0.95)^{*}\end{array}$ & $\begin{array}{c}<0.001 \\
0.01\end{array}$ \\
\hline CVD mortality & $\begin{array}{l}\text { Model } 1 \\
\text { Model } 2\end{array}$ & $\begin{array}{l}1.00 \\
1.00\end{array}$ & $\begin{array}{l}0.82(0.65-1.02) \\
0.85(0.67-1.10)\end{array}$ & $\begin{array}{l}0.60(0.46-0.77)^{*} \\
0.69(0.51-0.92)^{*}\end{array}$ & $\begin{array}{c}<0.001 \\
0.01\end{array}$ \\
\hline CVD events & $\begin{array}{l}\text { Model } 1 \\
\text { Model } 2\end{array}$ & $\begin{array}{l}1.00 \\
1.00\end{array}$ & $\begin{array}{l}0.90(0.75-1.08) \\
0.98(0.80-1.18)\end{array}$ & $\begin{array}{l}0.80(0.66-0.97)^{*} \\
0.93(0.75-1.15)\end{array}$ & $\begin{array}{l}0.03 \\
0.53\end{array}$ \\
\hline CHD events & $\begin{array}{l}\text { Model } 1 \\
\text { Model } 2\end{array}$ & $\begin{array}{l}1.00 \\
1.00\end{array}$ & $\begin{array}{l}0.93(0.73-1.17) \\
0.95(0.74-1.22)\end{array}$ & $\begin{array}{l}0.65(0.49-0.85)^{*} \\
0.74(0.55-0.99)^{*}\end{array}$ & $\begin{array}{l}0.002 \\
0.05\end{array}$ \\
\hline
\end{tabular}

Values are HRs (95\% CIs). Model 1: Age adjusted. Model 2: Adjusted for age, smoking, alcohol, social class, physical activity, body mass index, energy intake. $* P<0.05$.

1384 deaths, 553 CVD deaths, 895 CVD events and 488 CHD events occurred during the follow-up period. EDI and HDI were modestly correlated $(r=0.24, p<0.001)$. Men in the highest tertile of EDI scores had a significantly decreased risk of all-cause mortality, CVD mortality and CHD events after adjustment for confounders (all $p<0.05$ ). However, HDI was not significantly associated with any of the outcome measures.

In conclusion, EDI may be useful for assessing diet quality in older men as it appears to be a stronger predictor of CVD and all-cause mortality, compared to HDI. Efforts should be made to encourage older adults to adhere to EDI dietary recommendations.

1. Tourlouki E, Matalas AL, Panagiotakos DB (2009) Clinical interventions in aging 4, 319-30.

2. Kourlaba G, Polychronopoulos E, Zampelas A, Lionis C, Panagiotakos DB (2009) Journal of the American Dietetic Association 109(6), 1022-30.

3. Huijbregts P, Feskens E, Rasanen L, Fidanza F, Nissinen A, Menotti A et al. (1997) BMJ 315(7099), $13-7$. 